\title{
Universitarias en España: De los inicios a la actualidad*
}

\author{
University women in Spain: From the beginning to the current time
}

\author{
Universitárias na Espanha: Das origens à atualidade
}

\author{
Ana Guil Bozal ${ }^{1}$ \\ Universidad de Sevilla - España \\ Grupo de investigación HISULA - UPTC \\ anaguil@us.es \\ Consuelo Flecha García ${ }^{2}$ \\ Universidad de Sevilla - España \\ Grupo de investigación HISULA - UPTC \\ cflecha@us.es
}

Recepción: 09/03/2014

Evaluación: 06/08/2014

Aceptación: 24/11/2014

Artículo de reflexión

\section{RESUMEN}

En este artículo se ofrece un análisis de la la condición femenina no favorecían evolución cuantitativa de la presencia de ese tipo de decisiones, el objetivo es mujeres en las universidades españolas, desde las pioneras decimonónicas hasta dar a conocer el evidente y progresivo crecimiento en las aulas universitarias, el comienzo del nuevo milenio. Partiendo de la hipótesis de que el contexto social y la mentalidad generalizada acerca de primero de alumnas y más tarde de profesoras. La metodología utilizada ha partido de una revisión de la bibliografía

* Este articulo se interrelaciona con el proyecto "La Universidad en la capacitación de maestras rurales SGI 1034" y "Las educadoras en las Escuelas Normales en Colombia SGI 1293” desarrollado por el grupo HISULA - UPTC.

1 Doctora en Psicología Social por la Universidad de Sevilla, es catedrática de Escuela Universitaria en la misma Universidad, en la que imparte docencia desde 1980. Es responsable del Grupo de Investigación Género y Sociedad del Conocimiento, del Plan Andaluz de Investigación, Desarrollo e Innovación, siendo su especialidad el análisis de las barreras a la promoción profesional de las mujeres en la Universidad y en el Sistema de Ciencia y Tecnología. Su dirección profesional es: Departamento de Psicología Social, Facultad de Psicología, e integrante del grupo de investigación HISULA adscrito a la Universidad Pedagógica y Tecnológica de Colombia.

2 Doctora en Ciencias de la Educación por la Universidad Complutense de Madrid, es catedrática de Historia de la Educación en la Universidad de Sevilla. Desarrolla su docencia e investigación en temas relacionados con las trayectorias educativas de las mujeres, sobre las que ha publicado numerosos trabajos. Pertenece a varios proyectos de investigación sobre historia de las mujeres en las universidades y sobre distintos aspectos que favorecen dinámicas de coeducación en la enseñanza. Su dirección profesional es: Facultad de Ciencias de la Educación e integrante del grupo de investigación HISULA adscrito a la Universidad Pedagógica y Tecnológica de Colombia. 
disponible sobre esta temática, no muy abundante pero sí precisa en los datos e informaciones que ofrece; a continuación se ha buscado en las fuentes primarias que custodian los archivos universitarios y de la administración del Estado, en las series históricas de los anuarios estadísticos de alcance nacional e internacional. La síntesis, contraste y sistematización de todos los datos localizados nos ha permitido cubrir la finalidad prevista y ofrecer una imagen diacrónica de las mujeres que eligieron la universidad como proyecto primero de formación y luego profesional. La lectura de estas páginas permite comprobar algunas de las características de una cadencia temporal de largo plazo, casi ciento cincuenta años, y de la situación a la que se ha llegado a comienzos del siglo XXI, en que aún no se han logrado auténticas y reales oportunidades de igualdad en los ámbitos universitarios.

Palabras clave: Revista Historia de la Educación Latinoamericana, Universidad, Mujeres, Historia, Actualidad, España.

\section{ABSTRACT}

This article provides an analysis of the quantitative evolution of the women presence in Spanish universities, from the nineteenth-century pioneers to the beginning of the new millennium. Based on the assumption that the social context and the widespread mentality about the women's status did not look with favor to this kind of decisions, the objective is to publicize the clear and progressive increase in university classrooms, students first and then professors. The methodology used has begun with a review of the available literature on this subject, it is not very abundant but the data and the information provided is accurate; Below has been sought in the primary sources that keep the university files and the State administration, in the historical series of the Statistical Yearbooks from national and international reach. The synthesis, contrast, and systematization of all the located data has allowed us to cover the predicted purpose and to offer a diachronic picture of the women who chose the university project as training first and then professional. The reading of these pages allows checking some of the characteristics of a long-term temporary cadence, almost one hundred and fifty years, and the reached situation of the beginning of the twenty-first century where we did not achieve yet genuine and real equal opportunities in the academic environments.

Key words: History of Latin American Education Journal, University, Women, History, Current time, Spain.

\section{RESUMO}

Neste artigo se oferece uma análise da evolução quantitativa da presença de mulheres nas universidades espanholas, desde as pioneiras do século XIX até o começo do novo milênio. Partindo da hipótese de que o contexto social e a mentalidade generalizada acerca da condição feminina não favoreciam esse tipo de decisão, o objetivo é dar a conhecer o evidente e progressivo crescimento nas aulas universitárias, primeiro de alunas e mais tarde de professoras. A metodologia utilizada partiu de uma revisão da bibliografia 
disponível sobre esta temática, não muito abundante, porém precisa nos dados e informações que oferece; na sequencia se buscou nas fontes primárias que estão guardadas nos arquivos universitários e da administração do Estado, nas series históricas dos Anuários Estatísticos de alcance nacional e internacional. A síntese, contraste e sistematização de todos os dados localizados nos permitiu cobrir a finalidade prevista e oferecer uma imagem diacrônica das mulheres que elegeram a universidade como projeto primeiro de formação e, posteriormente, profissional. A leitura destas páginas permite comprovar algumas das características de uma cadência temporal de longo prazo, quase cento e cinquenta anos, e da situação a que se chegou no começo do século XX, em que ainda não se lograram autênticas e reais oportunidades de igualdade nos contextos universitários.

Palavras-chave: Revista História da Educação Latino-americana, universidade, mulheres, história, atualidade, Espanha.

\section{INTRODUCCIÓN}

En esta segunda década del siglo XXI que estamos viviendo, las mujeres universitarias continúan superando a los hombres entre el alumnado y suponen en torno al cuarenta por ciento del profesorado. Unos porcentajes que encontramos, con pequeñas diferencias, en casi todos los países del mundo con un nivel de desarrollo medio. Sin embargo, esta realidad convive con situaciones de discriminación de las niñas en los procesos educativos que no han desaparecido con el paso del tiempo, pues las vemos reproducidas aún en nuestros días, con mayor o menor incidencia de acuerdo con determinadas variables sociales ${ }^{3}$, por la persistencia de un modo de concebir las funciones de la población femenina apoyadas en la naturaleza biológica: en unas diferencias anatómicas a las que se atribuyen significados sociales y culturales que son fuentes de desigualdad ${ }^{4}$. Debido a estos mandatos de género, el itinerario recorrido por las generaciones que han precedido a las que hoy se encuentran con una situación cuantitativamente predominante en la universidad ha sido largo y sinuoso. Además de que no resulta suficiente, pues la meta de que todas las oportunidades de igualdad sean accesibles a mujeres y a hombres todavía no se ha producido.

Afirmamos esto debido a que, por ejemplo en la distribución de alumnas y de profesoras en los distintos niveles y modalidades de enseñanza y en las diversas

3 Consuelo Flecha García. "Because they’re girls. Overcoming inequality". Pedagogia oggi 1 (2013): 48-66.

4 Desde que la historiadora Joan W. Scott introdujo el género como categoría de análisis en los estudios históricos (recordemos su artículo, ya clásico, "Gender: A Useful Category of Historical Analysis" publicado en American Historical Review, en diciembre de 1986), su utilización ha marcado la investigación académica en historia de las mujeres. Ha permitido una mirada crítica tanto al pasado, con sus consecuencias acumulativas para mujeres y para hombres, como a los problemas que se mantienen en el presente sobre la construcción de lo femenino. 
áreas de conocimiento, se mantienen desequilibrios importantes de presencia, quizás como fruto de una subjetividad que entra en conflicto con los valores igualitarios en que han sido educadas ${ }^{5}$. Porque los puestos de mayor prestigio y salario siguen y seguirán estando mayoritariamente en manos masculinas mientras el funcionamiento de las organizaciones sea difícilmente compatible con otras dinámicas familiares y sociales, perdiendo de esta manera lo que las mujeres pueden aportar ${ }^{6}$. $\mathrm{O}$ porque criterios androcéntricos asignan menor valor a lo femenino y a lo feminizado, como se demuestra en las actividades donde ellas son mayoría o llegan a serlo. Unas características identificables en la realidad universitaria, donde tienen su respectivo efecto en los contenidos que se transmiten, en la investigación que se desarrolla, en los lugares que ocupan y en la incidencia real en su gestión y toma de decisiones ${ }^{7}$.

Ha pasado poco más de un siglo desde el reconocimiento a las mujeres del derecho a matricularse en las universidades españolas, exigiéndoles los mismos requisitos de entrada que a los hombres, aunque entonces casi ochenta alumnas habían pasado ya por las aulas de diferentes facultades; la pionera, en el curso 1872-1873 en la carrera de Medicina. Había algunos antecedentes de mujeres, de las que tenemos noticias más o menos documentadas, que asistieron durante el humanismo renacentista a las aulas universitarias y enseñaron en las Universidades de Alcalá de Henares y de Salamanca.

Fue en el año 1910 cuando se aprobó en España, mediante una Real Orden de 8 de marzo, que las mujeres dejaban de necesitar permiso ministerial para asistir como alumnas oficiales a las clases impartidas por los profesores. Con anterioridad, si no lo solicitaban, eran inscritas en la modalidad de alumnas no oficiales; es decir, acudían a la universidad únicamente los días en que se celebraran los exámenes de las asignaturas, las cuales debían haber preparado privadamente. De este modo se veían obligadas a superar, primero múltiples barreras sociales y sopesados acuerdos familiares antes de matricularse, y después la autorización ministerial, a lo que se sumaba el tener que ir acompañadas hasta las aulas y sentarse en un lugar especial al lado del profesor. $\mathrm{Y}$ al finalizar los estudios, tampoco se libraron de vetos a la hora de expedirles los títulos de licenciatura, de inscribirse en los Colegios Profesionales de Medicina o de Farmacia y de iniciar el ejercicio de la carrera estudiada.

5 Mónica Martínez y María Dolores Villuendas Giménez. "Las mujeres en la formación superior: elección de carrera versus estereotipos de género y neosexismos”. Cuestiones de género: de la igualdad y la diferencia 1 (2006): 87-112.

6 Un estudio pionero en España fue el de Ester Barberá et al., La promoción profesional de las mujeres en la Universidad (Valencia: Promolibro, 1998). Más recientemente ha analizado las relaciones entre género y liderazgo en las universidades Marita Sánchez Moreno et al., Mujeres dirigentes en la universidad: las texturas del liderazgo (Zaragoza: Prensas Universitarias de Zaragoza, 2009).

7 Pilar Ballarín Domingo. "Mujeres en el laberinto de cristal universitario". En Miradas desde la perspectiva de género: estudios de las mujeres, editado por Isabel de Torres Ramírez (Madrid: Narcea, 2005), 183-194. Ídem, "Docencia universitaria y conocimiento en torno al género. Resistencias, creencias y prejuicios”. Cuestiones de género: de la igualdad y la diferencia 8 (2013): 89-106. 
Vemos así cómo, desde sus inicios hasta la actualidad, las mujeres han tenido que recorrer un largo camino para poder acceder a la educación de más alto nivel y a su consiguiente profesionalización, camino no exento de dificultades, que aún no han ni mucho menos concluido pues, según nuestra hipótesis —que coincide con las de las principales estudiosas de estos temas-, las barreras a la profesionalización de las mujeres hunden sus raíces en posiciones patriarcales ante las que la evidencia de las cifras poco a nada tienen que hacer. La diferenciación social de los roles de género sobre la base biológica del sexo asocia desde tiempos ancestrales al varón con la obligación de ser el ganador de pan y a la mujer con las funciones reproductoras y cuidadoras, hipotecando con ello todo su tiempo e impidiéndole cualquier trabajo remunerado que no pase de un mero complemento al del varón; de ahí sus dificultades para llegar a ser profesionales universitarias.

Este es el panorama que nos disponemos a describir, utilizando para ello datos estadísticos históricos y actuales sobre la participación de las mujeres en la Universidad, procedentes de múltiples fuentes que iremos precisando y que, ciertamente, no han sido nada fáciles de recopilar dada la escasa conciencia existente sobre la importancia de desagregar los datos por sexo para conocer realmente la situación de las mujeres.

\section{Las primeras universitarias españolas}

En los siglos XV y XVI las crónicas históricas hablan de la presencia puntual de mujeres en las universidades de Salamanca y de Alcalá de Henares. Está documentada la de Teresa de Cartagena y Beatriz Galindo como alumnas en Salamanca, y las de Luisa de Medrano y Francisca de Lebrija como profesoras en Alcalá de Henares o Salamanca. Lucía de Medrano enseñaba Autores Clásicos Latinos y Francisca de Nebrija sustituía con aplauso y aprobación general a su padre en la cátedra de Retórica ${ }^{8}$. Pero seguramente la más conocida sea Beatriz Galindo, apodada "la Latina" — nombre que ostenta un barrio de la ciudad de Madrid-, por destacar como especialista de esa lengua en la Europa de aquellos momentos. No solo había estudiado Gramática en la Universidad de Salamanca en la segunda mitad del siglo XV, sino que llegó a ser la preceptora de latín de la reina Isabel la Católica y de sus hijas ${ }^{9}$.

Tres siglos después, en 1785, la madrileña María Isidra Guzmán de la Cerda obtuvo el grado de doctora en Filosofía y Letras Humanas en la Universidad de

\footnotetext{
8 Cristina Borreguero Beltrán. "Puellae doctae en las Cortes peninsulares”. Dossiers Feministes 15 (2010): 85-86.

9 Cristina Segura Graíno. "Beatriz Galindo. Ejemplo de humanista laica”. Miscelánea Comillas: Revista de Ciencias Humanas y Sociales 69 , 134 (2011): 293-304. María Isabel del Val Valdivieso. “Isabel la Católica y la educación”. Aragón en la Edad Media 19 (2006): $555-562$.
} 
Alcalá de Henares, después de ser autorizada por una Real Orden del Rey Carlos $\mathrm{III}^{10}$. Y en los años cuarenta del siglo XIX cuenta la tradición que Concepción Arenal (1820-1893), cubierta con una capa masculina, asistía a la Facultad de Derecho de la Universidad de Madrid ${ }^{11}$.

Todas ellas experiencias excepcionales y, en cuanto tales, sin posibilidad de servir de referencia a otras protagonizadas por mujeres de condición singular hasta que en 1872 se matricula María Elena Maseras Ribera en la Facultad de Medicina de la Universidad de Barcelona, inaugurando una etapa de continuidad de alumnas en las universidades españolas. En unos espacios académicos que desde ese momento no dejarán de tener matrículas femeninas, aunque la trayectoria sufriera una interrupción cuando, por una Real Orden de 1882, se impidió la matrícula de nuevas alumnas, aunque sí se les permitía finalizar la carrera a las que venían estudiando con anterioridad ${ }^{12}$.

Puesto que la prohibición no afectaba a las ya matriculadas, ese mismo año (1882) defendieron el doctorado en Medicina en la Universidad Central María Dolores Aleu Riera y Martina Castells Ballespí; en 1886 se licenciaron en Medicina Dolores Lleonart en Barcelona y María Luisa Domingo en Valladolid, y en Farmacia las hermanas Eloisa y María Dolores Figueroa Martí. Todavía terminaron otras tres alumnas en 1889: Concepción Aleixandre y Manuela Solís la carrera de Medicina en Valencia y Teresa de Andrés la de Filosofía y Letras en Barcelona ${ }^{13}$.

Ante las continuas demandas de algunas jóvenes, una nueva Real Orden de 11 de junio de 1888 acuerda admitirlas en los estudios de segunda enseñanza y superiores dependientes de la Dirección General de Instrucción Pública como alumnas de enseñanza no oficial en las condiciones ya señaladas: no poder asistir a las clases y, en caso de quererlo, teniendo que tramitar una consulta al Ministerio de Fomento del que dependían entonces los asuntos de instrucción pública. Un grupo de futuras estudiantes estaban atentas a las gestiones sobre esta reforma y esperaban tal decisión, lo cual posibilitó que inmediatamente, aprovechando el último plazo de matrícula del curso 1887-1888, se matricularan Ángela Carraffa de Nava y Matilde Padrós Rubió en la Facultad de Filosofía y Letras, estudiantes que llegaron a finalizar no solo la licenciatura sino también el doctorado ${ }^{14}$.

10 María Jesús Vázquez Madruga. María Isidra Quintina de Guzmán y la Cerda: la doctora de Alcalá (Alcalá de Henares: Centro Asesor de la Mujer, 1999). María-Milagros Rivera Garretas. La diferencia sexual en la historia (Valencia: Universidad de Valencia, 2005), 152-153.

11 María Carmen Sánchez Real. Concepción Arenal en su tiempo (Vigo: Ayuntamiento de Vigo, 1999).

12 Dándose cuenta de que eran cada vez más las mujeres que se animaban a cursar estudios universitarios, en 1882, recobrando la tradición patriarcal universitaria, y por una Real Orden de 16 de marzo, se suspendió "en lo sucesivo la admisión de las Señoras a la Enseñanza Superior".

13 Consuelo Flecha García. Las primeras universitarias en España (Madrid: Narcea, 1996), 227-229.

14 Ángela Carraffa de Nava defendió la tesis en 1892 y Matilde Padrós Rubió en 1893. Ídem. 
Después de veintidós años de un injusto trámite por el que muchas alumnas tuvieron que pasar, al fin la Real Orden de 8 de marzo de 1910 establecería que "se concedan, sin necesidad de consultar a la Superioridad, las inscripciones de matrícula en enseñanza oficial o no oficial solicitadas por las mujeres"15.

\section{El siglo XX: de excepcionalidad a mayoría}

Las casi ochenta jóvenes que realizaron estudios universitarios hasta la fecha de 1910 formaban parte de ese tipo de acontecimientos que suelen denominarse excepcionales y, como tales, utilizados por los canales de influencia para confirmar la normalidad de todo aquello que no seguía sus pasos. Pero esta singularidad empezó a desdibujarse cuando las autoridades reconocieron el derecho que tenían las mujeres a matricularse en las facultades universitarias cumpliendo los mismos requisitos exigidos a los hombres. No solo comenzó a aumentar el número de matrículas femeninas, sino que se produjeron variaciones en las carreras de estudio elegidas pues otra normativa del mes de septiembre de ese mismo año abrió a las mujeres la posibilidad de acceso a los concursos que el Ministerio de Instrucción Pública y Bellas Artes convocaba para ocupar plazas de profesorado en institutos de segunda enseñanza y en universidades y puestos técnicos en bibliotecas, archivos y museos.

Así, en el período que cierra el inicio de la Guerra Civil Española (1936), el número de mujeres estudiantes en el conjunto de las universidades españolas - pasaron de diez a doce en estos años - fue creciendo con rapidez, aunque todavía representaran una minoría dentro del total de alumnado y mas aún de la población femenina. Los datos recogidos en la Tabla 1 nos permiten conocer el avance de los números absolutos y de los porcentajes de alumnas sobre el total del alumnado.

15 Ana Guil Bozal. “1910-2010. Cien años buscando igualdad en la Universidad”. Aequalitas: Revista jurídica de igualdad de oportunidades entre mujeres y hombres 26 (2010): 16-19. Consuelo Flecha García. "Por Derecho Propio. Universitarias y Profesionales en España en torno a 1910”. Tabanque Revista Pedagógica 24 (2011): 157-174. Para una revisión bibliográfica de lo publicado sobre mujeres en las universidades españolas, ver Pilar Ballarín Domingo. "Entre ocupar y habitar. Una revisión historiográfica sobre mujeres y Universidad en España”. Arenal. Revista de Historia de Mujeres 17: 2 (2010): $223-254$. 
Tabla 1. Mujeres matriculadas y su porcentaje sobre el total del alumnado. 1910-1935

\begin{tabular}{|c|c|c|c|c|c|}
\hline Curso & N. $^{\circ}$ & $\%$ & Curso & N. $^{\circ}$ & $\%$ \\
\hline $1910-1911$ & 33 & 0,17 & $1929-1930$ & 1.744 & 5,2 \\
\hline $1916-1917$ & 177 & 0,56 & $1930-1931$ & 2.246 & 6,3 \\
\hline $1920-1921$ & 440 & 2,0 & $1931-1932$ & 2.026 & 6,0 \\
\hline $1923-1924$ & 898 & 3,5 & $1933-1934$ & 2.124 & 6,9 \\
\hline $1926-1927$ & 1.611 & 4,6 & $1935-1936$ & 2.588 & 8,8 \\
\hline
\end{tabular}

Fuente: Flecha, 2006: 481.

Partiendo de un proporción insignificante, el 0,17\%, terminaron alcanzando casi el nueve por ciento, subida a todas luces insuficiente pero valiosa en los años en que se produce. En las primeras décadas llegaron a un dos por ciento, en la tercera triplicaron ese valor, un seis por ciento, y en los años de la Segunda República la subida fue de otros casi tres puntos. Una evolución que también se produjo en la enseñanza secundaria, en la primaria y en los índices de alfabetización femenina, que se duplicaron de 1910 a 1940: pasaron de un treinta y ocho a un setenta por ciento ${ }^{16}$.

La Universidad de Madrid acogía al mayor número de estas jóvenes. Contaba con facultades de todas las disciplinas -Medicina, Farmacia, Filosofía y Letras, Ciencias y Derecho- y con el apoyo de dos residencias universitarias que garantizaban un alojamiento adecuado para las jóvenes que se desplazaban a la capital con esta finalidad, una condición mucho más importante en el caso de las mujeres que de los hombres, por el especial cuidado que recaía sobre ellas. La primera se inauguró en marzo de 1914 dentro del movimiento de Academias Teresianas, y la segunda en 1915, dependiendo de la Junta para Ampliación de Estudios, dirigida por la educadora y licenciada en Filosofía y Letras María de Maeztu Whitney (1881-1948) ${ }^{17}$.

La ampliación de campos profesionales vinculados a la administración educativa introdujo modificaciones en la prioridad de elección de las carreras universitarias. Medicina y Farmacia dejaron los primeros puestos mantenidos hasta entonces a Filosofía y Letras y a Ciencias que preparaban para la docencia de asignaturas de la segunda enseñanza, en especial del bachillerato, un ejercicio profesional mejor aceptado para las mujeres y donde encontraron de hecho muchas posibilidades de insertarse. La Tabla 2 va señalando la evolución y las

16 Narciso A. de Gabriel Fernández. “Alfabetización, Semialfabetización y Analfabetismo en España (1860-1991)”. Revista Complutense de Educación 8:1 (1997): 209.

17 Mercedes Montero. La conquista del espacio público. Mujeres españolas en la universidad (1910-1936) (Madrid: Minerva, 2009). 
fluctuaciones de su presencia en cada una de las facultades en el transcurrir de los sucesivos cursos académicos.

Tabla 2. Porcentajes sobre total de alumnas universitarias, por Facultades y Cursos Académicos

\begin{tabular}{|c|c|c|c|c|c|c|}
\hline Curso & Medicina & Farmacia & $\begin{array}{c}\text { Filosofía y } \\
\text { Letras }\end{array}$ & Ciencias & Derecho & Total \\
\hline $1872-1910$ & 48,0 & 24,7 & 20,5 & 4,1 & 2,7 & $100 \%$ \\
\hline
\end{tabular}

\begin{tabular}{|l|l|l|l|l|l|l|}
\hline $1914-1915$ & 22,2 & 17,8 & 30,0 & 27,8 & 2,2 & $100 \%$ \\
\hline $1916-1917$ & 19,2 & 16,4 & 26,6 & 37,2 & 0,6 & $100 \%$ \\
\hline $1918-1919$ & 18,7 & 21,3 & 25,8 & 32,9 & 1,3 & $100 \%$ \\
\hline
\end{tabular}

\begin{tabular}{|l|c|c|c|c|c|c|}
\hline $1920-1921$ & 14,6 & 26,2 & 23,2 & 34,2 & 1,8 & $100 \%$ \\
\hline $1923-1924$ & 13,3 & 32,2 & 24,2 & 28,3 & 2,0 & $100 \%$ \\
\hline $1926-1927$ & 8,1 & 27,7 & 25,6 & 36,4 & 2,2 & $100 \%$ \\
\hline
\end{tabular}

\begin{tabular}{|c|c|c|c|c|c|c|}
\hline $1928-1929$ & 11,7 & 49,1 & 22,4 & 11,7 & 5,1 & $100 \%$ \\
\hline $1930-1931$ & 9,7 & 43,2 & 25,1 & 14,9 & 7,1 & $100 \%$ \\
\hline $1932-1933$ & 8,9 & 25,1 & 35,9 & 17,1 & 13,0 & $100 \%$ \\
\hline
\end{tabular}

Fuente: Anuarios Estadísticos de España. Años correspondientes. Elaboración propia.

Estas oportunidades de trabajo abiertas en los institutos que impartían el bachillerato provocó el rápido crecimiento de las estudiantes de Filosofía y Letras y de Ciencias, facultades a las que se dirigieron para obtener un título que aseguraba un futuro prometedor. Del total de matrículas hasta el año 1910, las de Filosofía y Letras pasaron del tercer lugar al primero con un treinta por ciento en 1914; las de Ciencias se situaron como segundas con una llamativa subida, del cuatro al veintisiete por ciento. Medicina fue la facultad que más descendió proporcionalmente, y en Derecho siguieron representando un número insignificante de forma que las dos primeras no terminaron hasta 1921; fueron la valenciana María Ascensión Chirivella Marín y la madrileña María del Carmen López Bonilla, a las que se unió dos años más tarde, en 1923, la vallisoletana Luisa Cuesta Gutiérrez.

Continuaría el descenso relativo en Medicina y el incremento en Farmacia, carrera que ofrecía una salida profesional más fácil y aceptada para las mujeres; lo mismo que sucede en Filosofía y Letras respecto de Ciencias, que sigue incrementando los porcentajes, pues los estudios de esta facultad daban acceso a las oposiciones de entrada a plazas de bibliotecas, archivos y museos. Derecho avanza muy lentamente: apenas superó el dos por ciento ya que, por el contrario, no tenían entrada en la carrera judicial. 
El último tramo de esta Tabla 2 corresponde a los últimos años de la dictadura de Primo de Rivera y primeros de la Segunda República. Llaman la atención, por un lado, los porcentajes tan altos en Farmacia de 1928 a 1930 y, por otro, la disminución que se produce en los de Medicina, quizás éstos no ajenos a un ambiente que veía con reticencias la práctica de las mujeres de muchas especialidades médicas. Iniciado ya el cambio político republicano, vuelve a ser Filosofía y Letras la carrera donde se concentra un mayor número de alumnas, debido muy probablemente a la creación en 1932 de la Sección de Pedagogía en esa facultad, una especialidad con plan propio de entrada si se contaba con título de magisterio que muchas maestras aprovecharon para ampliar su formación y por haberse cerrado ese mismo año la Escuela de Estudios Superiores del Magisterio $^{18}$.

Y casi se duplican las alumnas de Derecho, de nuevo vinculado a un cambio legislativo, en este caso por el Real Decreto de 29 de abril de 1931, donde se recogía lo siguiente: "Será admitida la mujer a las oposiciones que se anuncien en lo sucesivo a Notarías y Registros de la Propiedad, y si obtuvieren plaza ingresarán en los respectivos Cuerpos, donde desempeñarán sus funciones en idénticas condiciones que el varón"19. Se trataba de unas salidas profesionales con prestigio a la que las jóvenes no quisieron renunciar; los datos demuestran su interés por formarse para poderlas desempeñar.

Como puede observarse, los datos experimentan una gran movilidad dependiendo de las oportunidades laborales a las que cada una de las facultades conducía. Los estudios de las mujeres del primer tercio del siglo XX, como había sucedido con las pioneras del XIX, tenían como objetivo no solo el conocimiento erudito de una determinada área científica, sino también una mirada de interés al mundo profesional en el que pudieran integrarse.

La Tabla 3 nos acerca a los números absolutos de cada facultad. Dan otra inmediatez a la proporcionalidad de los porcentajes y matizan algunas de las percepciones que provocan.

18 Antonio Molero Pintado. Escuela de Estudios Superiores del Magisterio (1909-1932): un precedente histórico en la formación universitaria del profesorado español (Guadalajara: Departamento de Educación de la Universidad de Alcalá de Henares, 1989).

19 Gaceta de Madrid 120, de 30 de abril de 1931: 407. 
Tabla 3. Número de Alumnas distribuidas por Facultades y Cursos académicos

\begin{tabular}{|l|c|c|c|c|c|c|}
\hline Curso & Medicina & Farmacia & $\begin{array}{l}\text { Filosofía y } \\
\text { Letras }\end{array}$ & Ciencias & Derecho & Total \\
\hline $1872-1910$ & 35 & 18 & 15 & 3 & 2 & 73 \\
\hline $1914-1915$ & 20 & 16 & 27 & 25 & 2 & 90 \\
\hline $1916-1917$ & 34 & 29 & 47 & 66 & 1 & 177 \\
\hline $1918-1919$ & 29 & 33 & 40 & 51 & 2 & 155 \\
\hline $1920-1921$ & 64 & 115 & 102 & 150 & 8 & 439 \\
\hline $1923-1924$ & 120 & 289 & 217 & 254 & 18 & 898 \\
\hline $1926-1927$ & 132 & 451 & 417 & 592 & 35 & 1.627 \\
\hline $1928-1929$ & 209 & 879 & 402 & 210 & 91 & 1.791 \\
\hline $1930-1931$ & 178 & 800 & 465 & 306 & 132 & 1.881 \\
\hline $1932-1933$ & 223 & 626 & 446 & 427 & 325 & 2.047 \\
\hline
\end{tabular}

Fuente: Anuarios Estadísticos de España. Años correspondientes. Elaboración propia.

Cantidades de dos dígitos, salvo en Derecho, en la segunda década del siglo que se multiplican a partir de 1920, de una manera llamativa en Farmacia, reseñable en Filosofía y Letras y en Ciencias, más prudente en Medicina y de cadencia muy lenta en Derecho hasta 1927, primer curso en que las matrículas pasan a ser el doble e inician un crecimiento que es más nítido en los dos últimos cursos de esta Tabla 3.

Las políticas que impulsaron el interés y las innovaciones en los procesos educativos y que alentaron a las mujeres a incorporarse más a todos los niveles de enseñanza darán como resultado un conjunto de transformaciones que empezarán en las aulas primarias y llegarán hasta los estudios universitarios ${ }^{20}$. Itinerarios que les fueron proporcionando una libertad y una autonomía desconocida por las generaciones anteriores y de efectos decisivos para las siguientes.

\section{El profesorado universitario}

Iniciarse en la docencia universitaria implicó una larga experiencia de éxitos académicos acumulados por parte de numerosas alumnas en las aulas universitarias. Pero hubo de esperar a que las implicaciones de los resultados fueran asimiladas por la sociedad y se entendiera que podían aspirar a ser profesoras en los institutos de segunda enseñanza e igualmente en la universidad, como ya lo eran en la enseñanza primaria las maestras.

20 Consuelo Flecha García. "La Segunda República, las Mujeres y la Educación". En Las Maestras de la República, editado por Elena Sánchez de Madariaga (Madrid: Catarata, 2012), 23-55. 
La que consideramos hasta ahora pionera en el estrado universitario se llamaba Luisa Cuesta Gutiérrez (1892-1962), que al finalizar la carrera en 1918 se estrenó como profesora auxiliar interina y gratuita de las asignaturas de Geografía Política y Descriptiva y de Paleografía en la Facultad de Filosofía y Letras de Valladolid; una excelente alumna que había finalizado la licenciatura en la Sección de Historia con sobresaliente y premio extraordinario ${ }^{21}$. Le había precedido Emilia Pardo Bazán, si bien en condiciones específicas; había sido nombrada en 1916 catedrática de una asignatura del período de doctorado de la Facultad de Filosofía y Letras de la Universidad de Madrid, por el Rey Alfonso $\mathrm{XIII}^{22}$, en virtud de sus méritos literarios, no como consecuencia de un itinerario de formación académica. El Ministro de Instrucción Pública Julio Burell la consideró en 1916 con cualidades innegables para ese cargo, aunque no recibió la conformidad del claustro de la Facultad de Filosofía y Letras ni de la Real Academia Española, una institución cuyos miembros ya le habían negado la entrada ${ }^{23}$. Se van sumando otras profesoras en todas las universidades, pero solo a partir del curso 1928-1929 figurarán en las estadísticas oficiales publicadas. Estas series periódicas nos permiten conocer los datos que recoge la Tabla 4.

Tabla 4. Profesorado universitario

\begin{tabular}{|c|c|c|c|c|}
\hline Curso & Mujeres & Hombres & Total & $\%$ Mujeres \\
\hline $1928-1929$ & 7 & 1.121 & 1.128 & $0.6 \%$ \\
\hline $1929-1930$ & 11 & 1.167 & 1.178 & $0,9 \%$ \\
\hline $1930-1931$ & 19 & 1.233 & 1.252 & $1,5 \%$ \\
\hline $1931-1932$ & 21 & 1.158 & 1.179 & $1,7 \%$ \\
\hline $1932-1933$ & 64 & 2.071 & 2.135 & $3,0 \%$ \\
\hline
\end{tabular}

Fuente: Flecha, 2013

Unos números que no podemos considerar definitivos, pues la búsqueda en los fondos documentales de cada universidad devuelve un número mayor. Las condiciones en que se producían los nombramientos de entrada en la docencia universitaria como ayudantes de clases prácticas, aprobados por el claustro de la facultad, daban invisibilidad administrativa fuera del centro concreto en que se producían. De hecho, la consulta realizada a los archivos históricos de varias universidades ha permitido identificar una serie de nombres de profesoras en cada curso académico, cuyo total es superior al que señalan las estadísticas.

21 Archivo Universidad de Valladolid (AUVA). Legajo 681-2 y Libro 306. Consuelo Flecha García. "Profesoras de Historia Medieval: mirando a los orígenes". En Impulsando la historia desde la historia de las mujeres, editado por Pilar Díaz (Huelva: Publs. Universidad de Huelva, 2012), 105-115.

22 Real Orden de 11 de marzo de 1916 creando en el Doctorado de la Facultad de Filosofía y Letras, Sección de Letras, la Cátedra de Literatura contemporánea de las Lenguas neo-latinas. Gaceta de Madrid 75, del 15 de marzo: 621. Real Decreto de 12 de mayo de 1916 nombrando Catedrático numerario (sic) de Literatura contemporánea de las Lenguas neo-latinas de la Universidad Central, a Da Emilia Pardo Bazán, Condesa de Pardo Bazán. Gaceta de Madrid 135, de 14 de mayo de 1916: 295.

23 Ángeles Quesada Novás. “Una meta alcanzada: La cátedra universitaria de Emilia Pardo Bazán”. La Tribuna 4 (2006): 56. 
Destacamos el salto cuantitativo en el curso 1932-1933, coincidiendo con el primer bienio de la Segunda República; se triplica el número absoluto y se duplica el porcentaje, coincidiendo con un substancial crecimiento del total de profesorado en el conjunto de las universidades. La localización de profesoras en cada universidad ${ }^{24}$ nos permite afirmar que siguieron aumentando, aunque no contamos con estadísticas oficiales para los años previos a la Guerra Civil de 1936. Sí sabemos que ninguna llegó a consolidar su puesto en la universidad, aunque lo intentaron; pero muchas de ellas concursaron a puestos docentes de los institutos de segunda enseñanza y a plazas de bibliotecas, archivos y museos. Estos ámbitos profesionales eran un camino más rápido para consolidar un puesto laboral y con él una autonomía económica y personal, pues los criterios del sistema de selección eran más objetivos.

Solo en la primera década del nuevo régimen político, los años cuarenta del siglo XX, la dictadura franquista, pudieron lograr algunas una estabilidad en la universidad presentándose a los concursos de plazas para profesoras adjuntas y obteniéndolas. Una ley de 1943 estableció el acceso a este cuerpo docente mediante concurso-oposición y, una vez aprobada, el rector hacía la propuesta al Ministerio de Educación Nacional, que lo hacía por cuatro años, prorrogables por otros cuatro ${ }^{25}$. Ya en los años cincuenta y sesenta, comenzaron a ocupar, mediante oposición, cátedras universitarias, un nivel administrativo al que solo llegaba una pequeña proporción del profesorado y que, en el caso de las mujeres, se fue produciendo con una llamativa lentitud. El prestigio social del profesorado universitario, el ambiente mayoritariamente masculino, la dedicación constante al estudio y a la investigación, el tener que viajar para participar en congresos, las publicaciones, etc., eran características y condiciones que costaba aceptar como apropiadas para las mujeres. Pero en el escalafón de cátedras de universidad, publicado por el Ministerio de Educación en 1974, figuraban once mujeres. La mayoría, siete, catedráticas de facultades de Filosofía y Letras, dos de facultades de Ciencias, una de Derecho y otra de Farmacia. La que rompe la unanimidad masculina a comienzos de los años cincuenta accede a una cátedra de Historia de la Pedagogía e Historia de la Pedagogía española. A ella se sumaron otras diez en la década de los sesenta. En la Tabla 5 aparecen los nombres de cada una, las disciplinas de su especialidad y las universidades en que ejercían. Un grupo muy pequeño distribuido en siete universidades, pero con una concentración en la de Santiago de Compostela, que contaba con cuatro de estas once catedráticas.

24 Hemos consultado los archivos históricos de las universidades de Granada, Madrid, Murcia, Salamanca, Santiago, Sevilla, Valencia, Valladolid y Zaragoza.

25 Ley de 29 de julio de 1943, sobre Ordenación de la Universidad Española, art. 62. Boletín Oficial del Estado 212, de 31 de julio. 
Tabla 5: Mujeres catedráticas hasta 1970

\begin{tabular}{|c|c|c|c|c|}
\hline Nombre & Año & Disciplina & Facultad & Universidad \\
\hline Galino Carrillo, Ángeles & 1953 & Historia de la Pedagogía & Filosofía y Letras & UCM \\
\hline $\begin{array}{l}\text { Linares Rodríguez, } \\
\text { Asunción } \\
\end{array}$ & 1961 & Paleontología & Ciencias & UGR \\
\hline Virgili Rodón, Carmen & 1963 & $\begin{array}{l}\text { Estratigrafía y } \\
\text { Geología Histórica }\end{array}$ & Ciencias & $\mathrm{UCM}$ \\
\hline Begúe Cantón, Gloria & 1965 & $\begin{array}{l}\text { Economía Política y } \\
\text { Hacienda Pública }\end{array}$ & Derecho & USAL \\
\hline Shaw Fairman, Patricia & 1966 & Lengua y Literatura Inglesa & Filosofía y Letras & UB \\
\hline Codoñer Merino, Carmen & 1966 & Lengua y Literatura Latinas & Filosofía y Letras & UOV \\
\hline Gómez Molleda, María Dolores & 1967 & $\begin{array}{l}\text { Historia Contemporánea } \\
\text { Universal y de España }\end{array}$ & Filosofía y Letras & USC \\
\hline Miralbes Bedera, $\mathrm{M}^{\mathrm{a}}$ del Rosario & 1967 & Geografía & Filosofía y Letras & USC \\
\hline Pérez Martín, María Jesús & 1970 & Lengua y Literatura Latinas & Filosofía y Letras & UVA \\
\hline Bobes Naves, María del Carmen & 1970 & $\begin{array}{l}\text { Gramática histórica de la } \\
\text { Lengua española }\end{array}$ & Filosofía y Letras & USC \\
\hline Fernández Otero, María del Pilar & 1970 & Fisiología animal & Farmacia & USC \\
\hline
\end{tabular}

Fuente: Escalafón de Catedráticos (sic) (1974)

Este acercamiento general diacrónico que hemos referido hasta aquí habla de un grupo de emprendedoras con voluntad y constancia, de una realidad de avance en la condición femenina que afectó a pocas mujeres hasta el último tercio del siglo $\mathrm{XX}$, pero que ilustra el origen de la situación a la que nos referimos en los siguientes apartados, es decir, a lo que ha ocurrido en el inicio del nuevo milenio, ayudándonos a valorar la evolución acaecida. A la vez, nos permite entender por qué está siendo tan difícil superar inercias e impedimentos para que las mujeres se muevan hoy con la libertad que les corresponde dentro de los ámbitos académicos.

\section{Las universitarias del siglo XXI}

Desde los años ochenta del siglo XX las estudiantes universitarias en España vienen superando a sus compañeros tanto en número de matrículas como de graduaciones; hay menos jóvenes matriculados, necesitan más años para finalizar la carrera, y la deserción es más frecuente entre ellos. En el curso 2011 - la fecha más reciente en que el Instituto Nacional de Estadística (INE) ha publicado resultados universitarios - eran mujeres aproximadamente el $54 \%$ del alumnado universitario y el 58,6\% de quienes se habían graduado. 
Pero no solo las mujeres han llegado a ser mayoría cuantitativa, sino que además son las que obtienen mejores rendimientos, como comenta Ana Crespo sobre datos de "La Universidad española en cifras 2011" y como igualmente muestran las últimas estadísticas europeas, que señalan mayores índices de abandono entre los varones y mayores porcentajes de graduaciones universitarias entre las mujeres, y ello como una constante en todos los países de la Unión Europea ${ }^{26}$.

También en másteres y doctorados las mujeres han empezado a superar cuantitativamente a los varones, si bien los porcentajes de lectura de tesis de hombres y mujeres oscilan en España entre el $44 \%$ y el 56\% en los últimos años, pues ciertamente se trata de una fase crítica en la carrera profesional universitaria de las mujeres.

Como muchos estudios describen y nosotras mismas hemos comprobado en diversas investigaciones ${ }^{27}$, no es casualidad el abandono de las mujeres en los años de lectura de la tesis, pues coinciden generalmente con los del inicio de la vida en pareja y la formación de una familia, situación frente a la que las mujeres - por la presión social de los roles de género- se encuentran en un dura disyuntiva al verse abocadas a tener que elegir entre familia-hijos y promoción profesional, algo que en absoluto sucede igual a sus compañeros varones que, por el contrario, ven aumentar su apoyo profesional y, en consecuencia, su tiempo de dedicación al trabajo remunerado y sus posibilidades de "hacer carrera", justo cuando comienzan a vivir en pareja o forman una familia.

Por eso, cuando analizamos las cifras del profesorado, las proporciones entre uno y otro sexo casi se invierten a partir de la tesis de doctorado, hasta disminuir los porcentajes de mujeres entre el profesorado al 38,7\%, situándose además, de manera inexplicable, mayoritariamente en las posiciones más bajas del escalafón, algo confirmado por los datos sobre docencia universitaria españoles y de casi todo el resto de países.

En el siguiente gráfico, Fig.1, sobre alumnado y profesorado universitario, representamos a modo de ejemplo y resumen la posición de mujeres y hombres en la universidad, en una instantánea de 2011 que ha cambiado muy poco en las últimas décadas.

\footnotetext{
26 Eurostat. The statistical office of the European Union.7 de marzo 2014.

27 Ver por ej. Ana Guil Bozal, Ana Solano y Manuela Álvarez. La situación de las mujeres en las universidades públicas andaluzas: P.D.I. y P.A.S (Sevilla: Consejo Económico y Social de la Junta de Andalucía, 2005).
} 
Figura 1: Alumnado y Profesorado Universitario España 2011

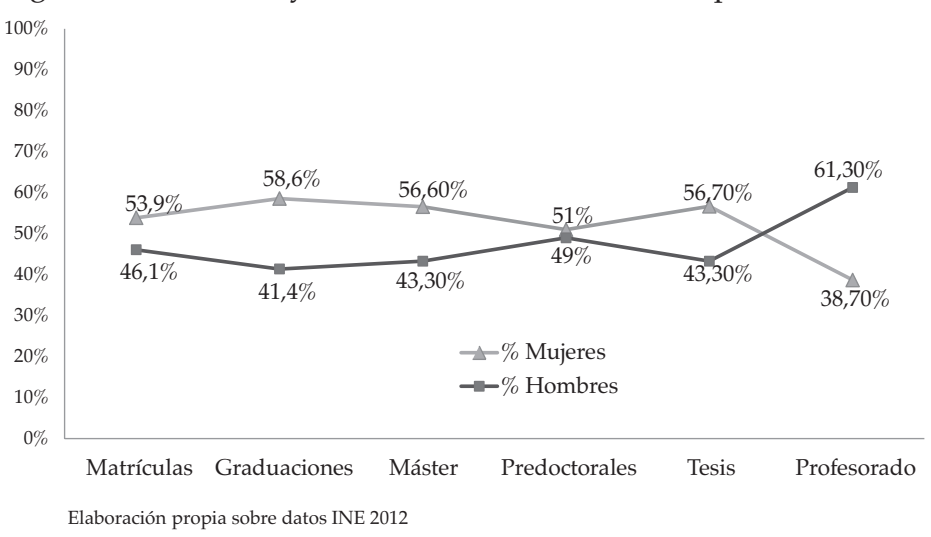

Como puede observarse, la realidad es que, pese al aumento progresivo de universitarias y los mejores resultados logrados, su trayectoria a lo largo del proceso académico que conduce al profesorado se mantiene en los primeros tramos y se invierte de manera incomprensible en el momento de ingresar en éste. El 43,3\% de hombres que han defendido la tesis pasan a ser el 61,3\% del profesorado; y del 56,7\% de mujeres doctoras solo permanece un 38,7\% de profesoras. Además, no es previsible que este desajuste vaya a solucionarse al menos a corto plazo, como se aprecia en la Fig. 2, que hemos elaborado para constatar la lenta evolución de los porcentajes de mujeres respecto del total de profesorado a lo largo de 30 años. Y menos aún si tenemos en cuenta que los retrocesos son posibles, máxime en épocas de crisis económica —como la actual en España-, en que las mujeres siempre llevaron históricamente las de perder.

Figura 2: Evolución del profesorado universitario en España

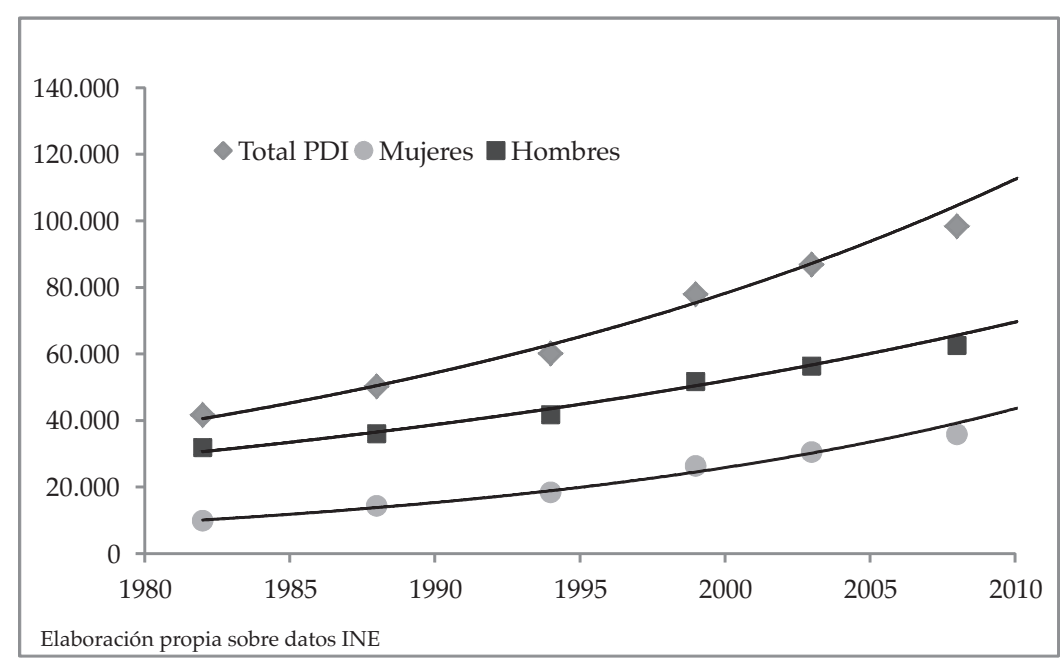


El profesorado se ha triplicado debido al crecimiento del alumnado en las universidades, una ocasión que tampoco ha podido ser aprovechada para que el porcentaje de mujeres dentro del cuerpo docente hubiera avanzado hasta llegar al menos a su constitución paritaria en España.

Y si nos centramos en las cátedras de universidad —la más alta categoría docente-, los porcentajes de mujeres, pese a la progresiva mejoría, continúan siendo desalentadores. Los datos de la Fig. 3 no pueden sino provocar ese sentimiento. El aumento de cátedras apenas redunda en la disminución del desajuste respecto de las mujeres, las cuales no dejan de comprobar las numerosas dificultades y circunstancias adversas que encuentran en el camino pautado, y tantas veces pactado, para obtenerlas.

Figura 3: Evolución de las Cátedras de Universidad en la primera década del siglo XXI

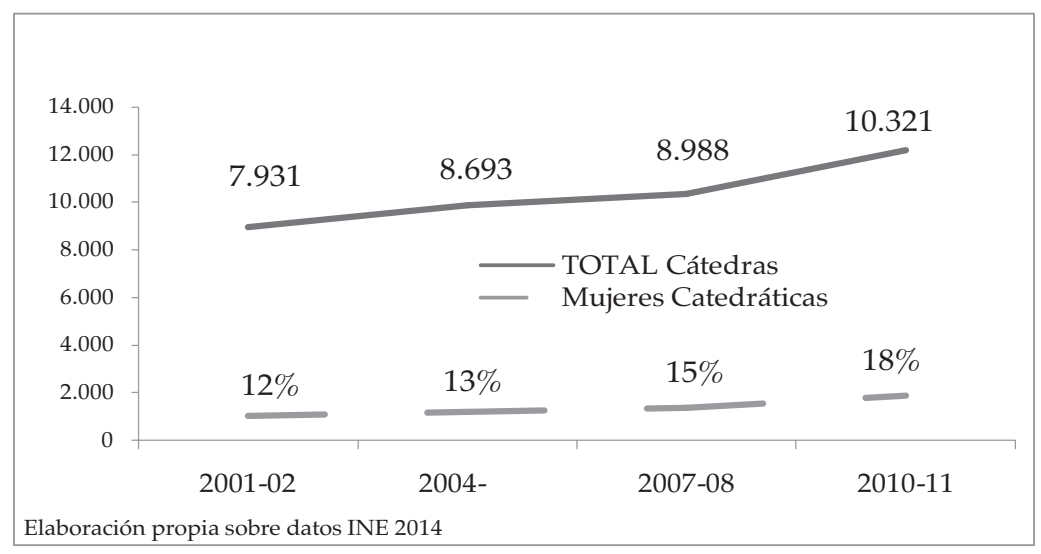

Una realidad que no solo afecta a España, pues en la comparación con los datos europeos el panorama tiene muchos puntos de coincidencia. En la Fig. 4 se puede apreciar esto fácilmente en el recorrido que presenta de la evolución de los porcentajes de estudiantes y de profesorado universitario, hombres y mujeres, desde el momento de acceso a la universidad hasta el más alto escalafón docente, datos referidos a un período de ocho años, del 2002 al 2010. 
Figura 4: Evolución de estudiantes y profesorado universitario en Europa

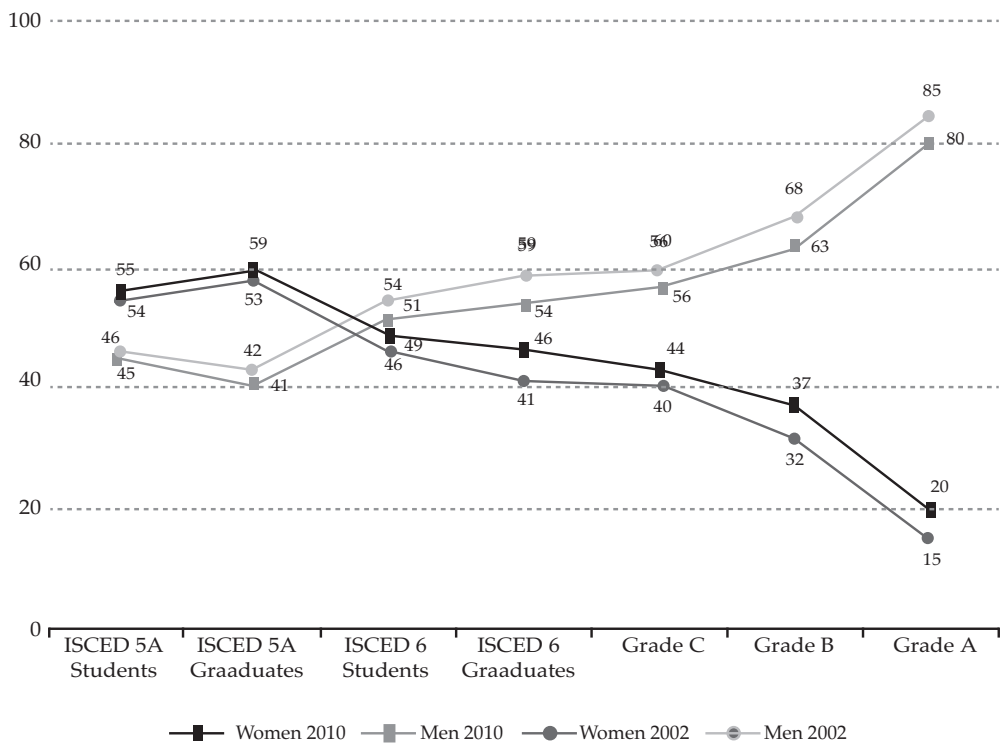

Fuente: She Figures 2012

La gráfica dibuja una tijera de mango corto porque enseguida confluye en un eje del que salen invertidas las dos líneas o filos, en una trayectoria de progresiva separación que el paso de los años no ha podido apenas modificar, sin dar prácticamente oportunidades para el equilibrio. Son muchas las preguntas que estas situaciones plantean, pero quizás podemos sintetizarlas, no sin actitud provocadora, en las siguientes: ¿la universidad prioriza el sexo de su profesorado por delante de la excelencia de la formación?, ¿dónde han quedado sus principios humanistas y racionalistas?, ¿acaso las mujeres no formamos parte del género humano?

\section{CONCLUSIÓN}

El indudable avance de las mujeres en los ámbitos universitarios desde sus inicios hasta la actualidad no ha podido desterrar la ancestral supremacía de los hombres, los cuales continúan ostentando el poder de enseñar, organizar y decidir, pese a su efectiva minoría cuantitativa entre el alumnado y a los mejores resultados académicos de las mujeres. Así se impone la realidad de que las posiciones más altas del escalafón, los puestos mejor remunerados y los de mayor visibilidad y reconocimiento público siguen siendo espacios vetados para la mayoría de las profesionales que han de conformarse con posiciones intermedias, pese a su demostrada valía. 
Mientras tanto, observan cómo ascienden los colegas hombres, junto a solo una exigua minoría de mujeres a las que consienten su llegada hasta posiciones ocupadas prioritariamente por ellos, con frecuencia para cubrir el expediente al hilo de una nueva conciencia sobre la equidad y prefiriendo a las que no rompan el orden patriarcal. Y no es ninguna exageración este modo de percibir pues, a la vista de los datos, ciertamente nuestras catedráticas y no digamos nuestras rectoras universitarias, después de varios siglos y pese a las características ya señaladas, siguen representando situaciones de excepción y, en cuanto tal, no generalizables.

La magnífica Ley de Igualdad española y las subsiguientes leyes derivadas no han conseguido superar los férreos techos de cristal que impiden a las mujeres ocupar, en igualdad de condiciones con los hombres, las posiciones universitarias que, además, les corresponderían doblemente por su ya mayor formación.

Y no se trata de un problema relacionado con la tardía incorporación de las mujeres a la universidad, pues ya han superado con creces esa ausencia multicentenaria. Más bien es un reflejo de nuestra sociedad patriarcal, que permea a sus instituciones todas y a cada una de las estructuras. La universidad no es ninguna excepción a esa regla androcéntrica.

Sin embargo, y desde una posición tal vez utópica, no podemos dejar de plantear que las universidades tienen la obligación de educar en la igualdad y que en consecuencia tendrían que ser referencia y ejemplo, de primera mano, de la participación equitativa de alumnado y profesorado en todas sus dinámicas de funcionamiento. Y no significa que muchas mujeres quieran ocupar los mejores puestos por reconocerse preparadas porque desean tener capacidad de decidir; simplemente aspiran a promocionar profesionalmente, a tener la opción de ocupar de manera paritaria las posiciones que les corresponden.

Quieren, en definitiva, que sea una realidad la democracia, la justicia social y la práctica cotidiana de todos los derechos de ciudadanía también para la otra mitad de la población, relegada en el proyecto de Estados modernos, esto es, para las mujeres. 


\section{FUENTES}

Gaceta de Madrid 120, de 30 de abril de 1931: 407.

Gaceta de Madrid 75, del 15 de marzo: 621.

Gaceta de Madrid 135, de 14 de mayo de 1916: 295.

Ley de 29 de julio de 1943, sobre Ordenación de la Universidad Española, art. 62. Boletín Oficial del Estado 212, de 31 de julio.

Real Orden de 11 de marzo de 1916 creando en el Doctorado de la Facultad de Filosofía y Letras, Sección de Letras, la Cátedra de Literatura contemporánea de las Lenguas neo-latinas.

Real Decreto de 12 de mayo de 1916 nombrando Catedrático numerario (sic) de Literatura contemporánea de las Lenguas neo-latinas de la Universidad Central, a D Emilia Pardo Bazán, Condesa de Pardo Bazán.

\section{REFERENCIAS}

Ballarín Domingo, Pilar. "Mujeres en el laberinto de cristal universitario". En Miradas desde la perspectiva de género: estudios de las mujeres, editado por Isabel de Torres Ramírez. Madrid: Narcea, 2005, 183-194.

Ballarín Domingo, Pilar. “Entre ocupar y habitar. Una revisión historiográfica sobre mujeres y Universidad en España”. Arenal. Revista de Historia de Mujeres 17: 2 (2010): 223-254.

Ballarín Domingo, Pilar. "Docencia universitaria y conocimiento en torno al género. Resistencias, creencias y prejuicios". Cuestiones de género: de la igualdad y la diferencia 8 (2013): 89-106.

Barberá, Ester et al. La promoción profesional de las mujeres en la Universidad. Valencia: Promolibro, 1998. Más recientemente ha analizado las relaciones entre género y liderazgo en las universidades Marita Sánchez Moreno et al., Mujeres dirigentes en la universidad: las texturas del liderazgo. Zaragoza: Prensas Universitarias de Zaragoza, 2009. 
Borreguero Beltrán, Cristina. “Puellae doctae en las Cortes peninsulares". Dossiers Feministes 15 (2010): 85-86.

Crespo, Ana. Las universitarias son más y tienen mejor rendimiento. AmecoPress. Información paralaIgualdad, 2011.http: / / www.amecopress.net/ spip.php?article6079 (acceso: 27/11/2013).

Eurostat. The statistical office of the European Union, 7 marzo 2014.

Flecha García, Consuelo. Las primeras universitarias en España: 1872-1910. Madrid: Narcea, 1996.

Flecha García, Consuelo "Profesoras en la Universidad. El tránsito de las pioneras en España". Arenal 17: 2 (2010): 255-297.

Flecha García, Consuelo. “Por Derecho Propio. Universitarias y Profesionales en España en torno a 1910". En Tabanque Revista pedagógica 24 (2011): 157-174.

Flecha García, Consuelo. “La Segunda República, las Mujeres y la Educación”. En Las Maestras de la República, editado por Sánchez de Madariaga, Elena. Madrid: Catarata, 2012: 23-55.

Flecha García, Consuelo. "Profesoras de Historia Medieval: mirando a los orígenes". En Impulsando la historia desde la historia de las mujeres, editado por Díaz, Pilar. Huelva: Publs. Universidad de Huelva, 2012.

Flecha García, Consuelo. "Because they're girls. Overcoming inequality". Pedagogia oggi 1 (2013): 48-66.

Flecha García, Consuelo. "Ganando espacios en la universidad española en la primera mitad del siglo XX". En Comiendo del fruto prohibido. Mujeres, ciencia y creación a través de la historia, editado por Del Val, María Isabel-Martínez Quinteiro, Esther. Madrid: Icaria, 2014.

Gabriel Fernández, Narciso A. de. "Alfabetización, Semialfabetización y Analfabetismo en España (1860-1991)". Revista Complutense de Educación 8:1 (1997): 199-232.

Guil Bozal, Ana. Análisis del número de mujeres y hombres de la USE que solicitan y disfrutan la baja por maternidad/paternidad, acogimiento, adopción, lactancia y excedencia después de un nacimiento, así como su repercusión económica. Unidad para la Igualdad. Universidad de Sevilla, 2011. 
Guil Bozal, Ana. "1910-2010. Cien años buscando igualdad en la Universidad". Aequalitas: Revista jurídica de igualdad de oportunidades entre mujeres y hombres 26 (2010): 16-19.

Guil Bozal, Ana. Techos de cristal en la Universidad de Sevilla. 2009. http: / / www.amites.org/assets / files/techos_cristal_anaguil.pdf

Guil Bozal, Ana. "Mujeres y Ciencia: Techos de Cristal". ECCOS Revista de Investigación Científica, Universidade Nove de Julho, Brasil. www.uninove.br/ publicaçoês (2008): 213-232.

Guil Bozal, Ana. "Docentes e investigadoras en las universidades españolas: visibilizando techos de cristal" Revista de investigación Educativa 25:1 (2007).

Guil Bozal, Ana. "Barreras al desarrollo profesional de las mujeres en la Universidad". En Ciencia, Tecnología y Género en Iberoamérica, editado por Pérez Sedeño, Eulalia et al. Madrid: Consejo Superior de Investigaciones Científicas, 2006: 99-108.

Guil Bozal, Ana. “Techos de cristal universitarios: buscando, visibilizando e interpretando datos". En Democracia, feminismo y Universidad en el siglo XXI, editado por Maqueira, V. et al. Madrid: Instituto Universitario de Estudios de la Mujer, Universidad Autónoma de Madrid, 2005: 187-198.

Guil Bozal, Ana. "Glass ceiling in the University". En Catching the Future: Women and Men in Global Psychology, editado por R. Roth, L. Lowestein, D. Trent. Lengerich (Germany): Pabst Science Publishers, 49525, 2003: 119-127.

Guil Bozal, Ana. "El viaje de Penélope. Sobre las vicisitudes de las docentes universitarias". El Telar de Ulises $n^{\circ}$ 2/Mujeres y Relidad Social 11. 2002. www.us.es/ bibemp/ulises/ulises2 / Penelope.htm

Guil Bozal, Ana; Solano, Ana y Álvarez, Manuela. La situación de las mujeres en las universidades públicas andaluzas: P.D.I. y P.A.S. Sevilla: Consejo Económico y Social de la Junta de Andalucía, 2005.

Guil Bozal, Ana; Hou, JingRong y Jiménez, Yirsa. “Glass ceiling in the Universitycross cultural perspectives". En Making a Differences in the Life of Others, editado por Dayan et al. Aachen (Germany): Shaker Verlag, 2006: 139-148.

Instituto Nacional de Estadística. Estadística de la Enseñanza Universitaria en España. Curso 2010/2011. Madrid: Instituto Nacional de Estadística, 2012. 
Martín García, Teresa. Científicas en cifras. Coordinadora: Inés Sánchez de Madariaga. Unidad de Mujeres y Ciencia. Ministerio de Economía y Competitividad. http:/ / www.mujeryciencia.es/2012/01/10/ cientificas-en-espana-en-cifras-2011 / , 2011.

Martínez, Mónica y Villuendas Giménez, María Dolores. “Las mujeres en la formación superior: elección de carrera versus estereotipos de género y neosexismos". Cuestiones de género: de la igualdad y la diferencia 1 (2006): 87-112.

Molero Pintado, Antonio. Escuela de Estudios Superiores del Magisterio (1909-1932): un precedente histórico en la formación universitaria del profesorado español. Guadalajara: Departamento de Educación de la Universidad de Alcalá de Henares, 1989.

Montero, Mercedes. La conquista del espacio público. Mujeres españolas en la universidad (1910-1936). Madrid: Minerva, 2009.

Pérez Sedeño, Eulalia; Guil Bozal, Ana et al. La situación de las mujeres en el sistema científico español y su contexto internacional. www.campus-oei.org/salactsi/EA20030031.pdf, 2004.

Quesada Novás, Ángeles. “Una meta alcanzada: La cátedra universitaria de Emilia Pardo Bazán”. La Tribuna 4 (2006): 43-81.

Rivera Garretas, María-Milagros. La diferencia sexual en la historia. Valencia: Universidad de Valencia, 2005.

Sánchez Real, María Carmen. Concepción Arenal en su tiempo. Vigo: Ayuntamiento de Vigo, 1999.

Scott, Joan W. "Gender: A Useful Category of Historical Analysis". American Historical Review 91:5 (Dec. 1986): 1053-1075.

Segura Graíño, Cristina. “Beatriz Galindo. Ejemplo de humanista laica”. Miscelánea Comillas: Revista de Ciencias Humanas y Sociales 69:134 (2011): 293-304.

She figures 2012. Gender in Research and Innovation. Statistics and Indicators. European Commission. http:/ / ec.europa.eu/research / science-society / document_library / pdf_06/ she-figures-2012_en.pdf

Val Valdivieso, María Isabel de. "Isabel la Católica y la educación”. Aragón en la Edad Media 19 (2006): 555-562.

Vázquez Madruga, María Jesús. María Isidra Quintina de Guzmán y la Cerda: la doctora de Alcalá. Alcalá de Henares: Centro Asesor de la Mujer, 1999. 


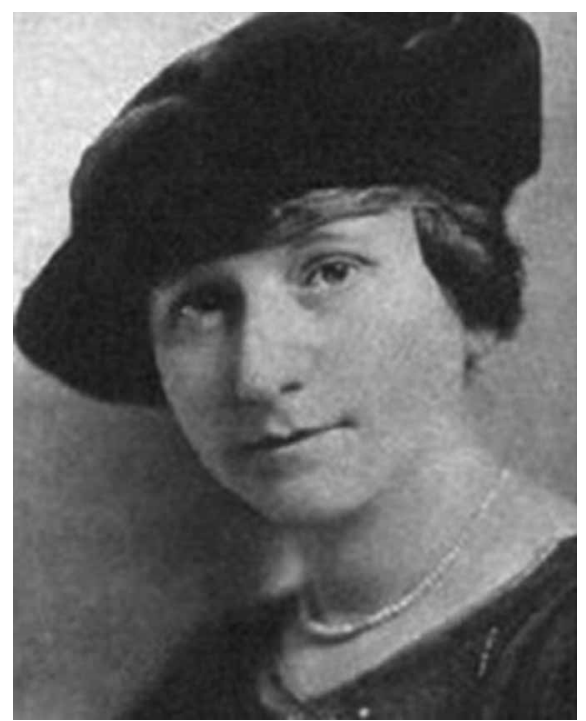

María de Maeztu, directora de la Residencia de Señoritas creada en 1915.

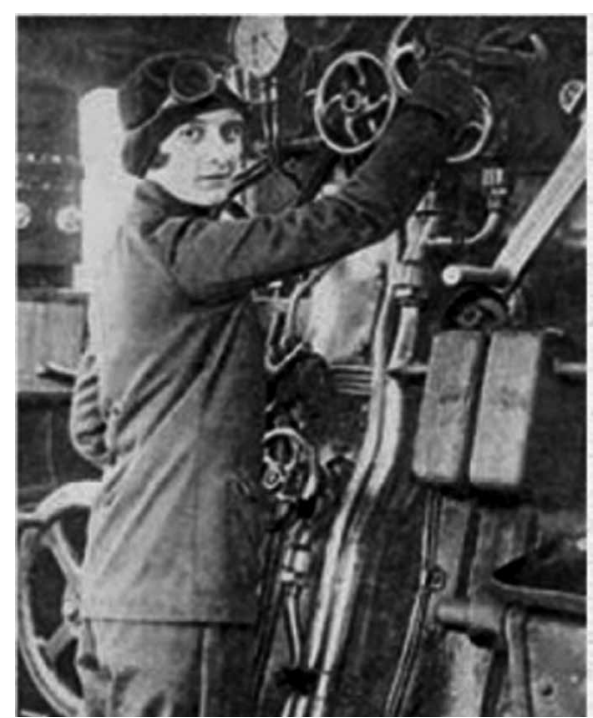

Pilar Careaga Besabe, primera mujer ingeniera de España, graduada en 1929 en la Escuela de Ingenieros Industriales de Madrid.

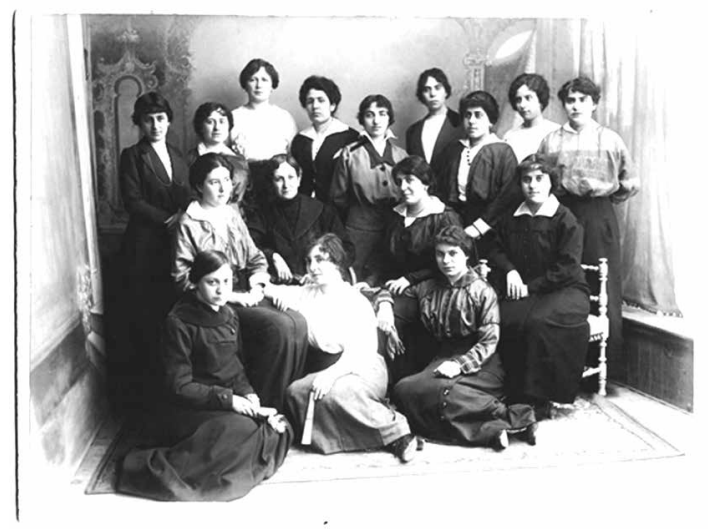

Primeras alumnas de la

Residencia Universitaria Teresiana en 1914.

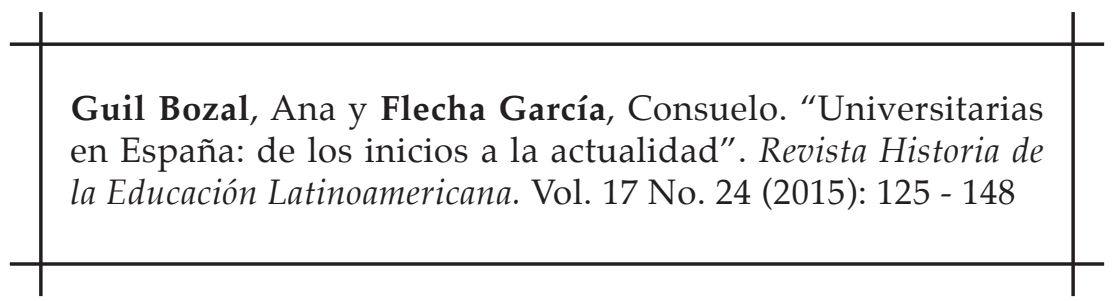

